\title{
Modelling and Simulation of a Paraglider Flight
}

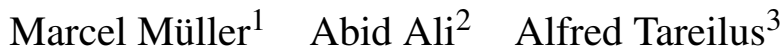 \\ ${ }^{1}$ Faculty of Electrical Engineering, University of Applied Sciences Würzburg-Schweinfurt, D-97421 Schweinfurt, \\ Germany, marcel.mueller.3estudent. fhws.de \\ ${ }^{2}$ Faculty of Electrical Engineering, University of Applied Sciences Würzburg-Schweinfurt, D-97421 Schweinfurt, \\ Germany, abid.ali@fhws.de \\ ${ }^{3}$ Gleitschirmfreunde Schweinfurt e.V., D-97506 Grafenrheinfeld, Germany, alfred.tareilus @web. de
}

\begin{abstract}
A simulation model of a paraglider flight is presented in this paper. The dynamics of the paraglider trajectory, as well as its twist angle are governed by a complex interplay between gravity and drag force. Differential equations describing this dynamical behaviour are developed by balancing all the forces and torques acting on the system. The developed model allows to compare different flight situations and find out optimal values of the parameters, which may be used in further steps for example optimization of the towing process. Simulation results of the paraglider launch process using a vehicle unreeling winch and subsequent gliding under different wind conditions are compared with real data.
\end{abstract}

Keywords: paraglider, gliding flight, towing process, aerodynamics, simulation, modelling

\section{Introduction}

In the 1960s, a hype about paragliding started in Europe. The performance of gliders increased significantly and the crafts were easier to control due to advanced designs. This was a major step towards making the flight safer (Currer, 2011). However, the development of modern paragliders, the ones we know today, needed a long time. There are some competitions in different disciplines as well, whereby aerial acrobatics like spiral dives or full stalls are often performed (Whittall, 2010). Most pilots drive to big mountains like the Alps to enjoy paragliding.

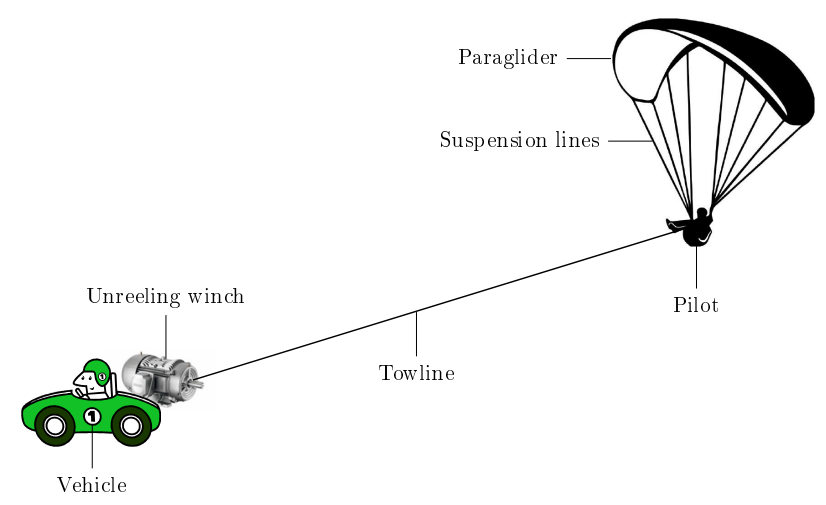

Figure 1. Winch tow launch.
Potential energy or a start altitude is transformed into kinetic energy to bridge distances as long as possible. This process is called "gliding flight". But there is an alternative method for paragliding as well. The so called winch tow launch can also be used in lowlands (see Figure 1). With the help of an unreeling winch mounted on the back of a vehicle the pilot is pulled into the air. Various parameters can be influenced during this process to reach the best possible outcome. A simulation model of the towing procedure can be helpful for optimisation of the process.

The standard reference written by Janssen et al. (2013) for paragliders and hang gliders provides guidelines about different security measures to prevent hazard situations. Consequently, it is an ideal guide for trainee pilots as well as experienced gliders. Whittall (1995) introduced basic flying techniques and presented additional information about equipment, weather and soaring. Over the years, the aerodynamics and flight mechanics of paragliders improved continuously and the fundamentals of fluid mechanics as well as the involved physical processes are nowadays well understood (Oertel, 2005). A detailed description of the effective forces acting on the paraglider and the pilot during a gliding flight can be visualised with the help of a free body diagram (Voigt, 2003). This free body diagram can be augmented in order to describe the so-called towing process (see Figure 1), whereby the pilot starts from the ground and is lifted into the air (Fahr, 1992). A polar curve, also named as Lilienthal Curve (Karbstein, 1996), describes the ratio of sink velocity to air speed in $\mathrm{x}$-direction for a specific glider under different operating conditions. It allows, as a decisive advantage, to predict the glider's performance (Currer, 2011).

In spite of all the improvements and understanding of physical phenomenon and parameters, there is no complete model available, which could be employed to simulate the dynamical behaviour of the system. The aim of this contribution is to derive such a mathematical model. It could be used to simulate the system behaviour in towing and gliding states. This could lead on one hand to analyse the performance, safety and stability of paragliders and to optimise the towing process on the other hand. This paper is organised as follows. A complete mathematical model of the system is derived in section 2. How this model can be parametrised, is discussed in section 3. Sec- 
tion 4 presents some simulation results, while conclusions and a brief outlook are given in section 5 .

\section{Modelling}

In order to develop the model a simplified free body diagram of the whole system is drawn in Figure 4. The mass of the paraglider $m_{g}$ and the mass of the pilot $M_{p}$ are assumed to be connected to each other rigidly. The aim is to derive differential equations describing translational and rotational dynamics of the system. A coordinate system "CS" is displayed next to the figures to define the positive axes and angles.

\subsection{Centre of Gravity}

The centre of gravity $G$ is the point at which the sum of all torques due to gravitational forces equals zero. The two torques involved are the torque due to the weight of the pilot and the torque due to the weight of the paraglider. Consequently, the distance $l_{M G}$ can be determined (see equation (2)). With this information, $l_{G m}$ is calculable with the help of the length of the suspension lines $l_{\text {line }}$ as well (equation (3)). The line length is known from the data sheet provided by a paraglider manufacturer which is used as a reference glider in the simulation.

$$
\begin{aligned}
M_{p} \cdot g \cdot l_{M G} & =m_{g} \cdot g \cdot\left(l_{\text {line }}-l_{M G}\right) \\
\Leftrightarrow l_{M G} & =\frac{m_{g} \cdot l_{\text {line }}}{M_{p}+m_{g}}=\frac{m_{g} \cdot l_{\text {line }}}{m_{t}} \\
l_{G m} & =l_{\text {line }}-l_{M G}
\end{aligned}
$$

\subsection{Aerodynamic Forces}

Aerodynamics deal with forces caused by air flow around an object. Typical forces and angles of a paraglider in static conditions are shown in Figure 2. The notational symbols are explained in Table 1 . The weight $F_{W, t}$ points

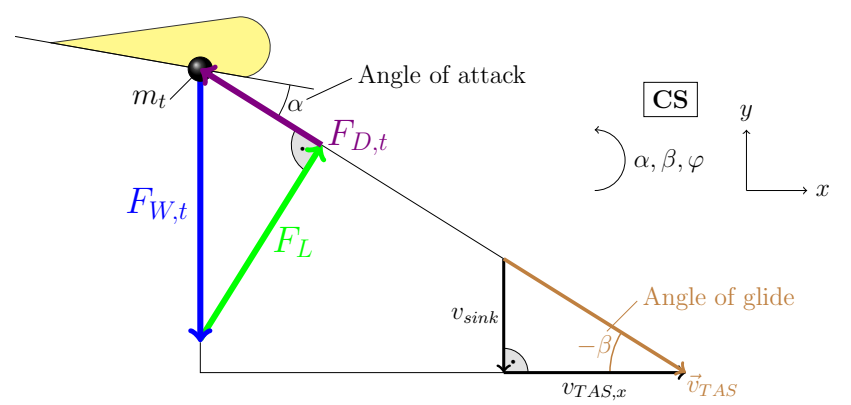

Figure 2. Aerodynamic forces.

vertically downwards and is compensated by the total aerodynamic force which is the resultant of the lift force $F_{L}$ and the drag force $F_{D, t}$. They are perpendicular to each other whereby $F_{L}$ creates the lift to glide through the air. The drag force $F_{D, t}$ is directed contrary to the direction of movement. Drag is caused by friction between paraglider and air and converts a part of the energy into losses. The
Table 1. Notations.

\begin{tabular}{ll} 
Symbol & Description \\
\hline \hline$F_{D, t}$ & Drag force \\
$F_{L}$ & Lift force \\
$F_{W, t}$ & Weight \\
$T_{D, g}$ & Paraglider torque caused by \\
& drag force \\
$T_{D, p}$ & Pilot torque caused by drag \\
& force \\
$T_{L}$ & Torque caused by lift force \\
$T_{r e s}$ & Resultant torque clockwise \\
$T_{\text {start }}$ & Start torque
\end{tabular}

half density of the air $\frac{\rho_{a i r}}{2}$ multiplied by the squared true air velocity $\left(v_{T A S}\right)^{2}$ yields the dynamic pressure $p_{\text {dynamic }}$ of the air. The total drag force of a paraglider $F_{D, t}$ is equal to the dynamic pressure $p_{\text {dynamic }}$ multiplied by the projected area $A_{\text {project }}$ and the drag coefficient $c_{d, g}$ of the paraglider (Anderson, 2005).

$$
F_{D, t}=c_{d, g} \cdot A_{\text {project }} \cdot \underbrace{\frac{\rho_{\text {air }}}{2} \cdot\left(v_{T A S}\right)^{2}}_{p_{\text {dynamic }}}
$$

Figure 2 displays how the total drag force $F_{D, t}$, under the assumption of static conditions, alternatively can be determined. It means that no acceleration force has an affect on the system. The drag force $F_{D, t}$ equals the total weight $F_{W, t}$ multiplied by the sine of the negative angle of glide $\sin (-\beta)$, which in turn is the sink velocity $v_{\text {sink }}$ divided by the true air speed $v_{T A S}$.

$$
F_{D, t}=F_{W, t} \cdot \sin (-\beta)=F_{W, t} \cdot \frac{v_{\text {sink }}}{v_{T A S}}
$$

Equating equation (4) with equation (5)

$$
\begin{aligned}
& c_{d, g} \cdot \frac{\rho_{\text {air }}}{2} \cdot A_{\text {project }} \cdot\left(v_{T A S}\right)^{2}=F_{W, t} \cdot \frac{v_{\text {sink }}}{v_{T A S}} \\
\Leftrightarrow & c_{d, g}=\frac{F_{W, t} \cdot 2}{A_{\text {project }} \cdot \rho_{\text {air }}} \cdot \frac{v_{\text {sink }}}{\left(v_{T A S}\right)^{3}}
\end{aligned}
$$

The lift force $F_{L}$ follows the same rule as the drag force (Anderson, 2005). The dynamic pressure $p_{\text {dynamic }}$ multiplied by the projected square $A_{\text {project }}$ and the lift coefficient $c_{l}$ is equal to the lift force.

$$
F_{L}=c_{l} \cdot A_{\text {project }} \cdot \underbrace{\frac{\rho_{\text {air }}}{2} \cdot\left(v_{T A S}\right)^{2}}_{p_{\text {dynamic }}}
$$

It is clear from Figure 2 that the lifting force $F_{L}$ equals the total weight $F_{W, t}$ multiplied by the cosine of the negative angle of glide $\cos (-\beta)$ which is equal to the ratio of the horizontal velocity $v_{T A S, x}$ and the true air speed $v_{T A S}$.

$$
F_{L}=F_{W, t} \cdot \cos (-\beta)=F_{W, t} \cdot \frac{v_{T A S, x}}{v_{T A S}}
$$


Equating equation (8) with equation (9)

$$
\begin{aligned}
& c_{l} \cdot \frac{\rho_{\text {air }}}{2} \cdot A_{\text {project }} \cdot\left(v_{T A S}\right)^{2}=F_{W, t} \cdot \frac{v_{T A S, x}}{v_{T A S}} \\
\Leftrightarrow & c_{l}=\frac{F_{W, t} \cdot 2}{A_{\text {project }} \cdot \rho_{\text {air }}} \cdot \frac{v_{T A S, x}}{\left(v_{T A S}\right)^{3}}
\end{aligned}
$$

\subsection{Definition of Angles}

The tangent of the glide angle $\beta$, located between horizontal line and direction of movement $v_{T A S}$ (see Figure 3), is calculated by the ratio of the air speed in y-direction to the air speed in $\mathrm{x}$-direction (equation (12)).

$$
\beta=\arctan \left(\frac{v_{T A S, y}}{v_{T A S, x}}\right)
$$

The angle of attack $\alpha$ is the angle at which the airflow meets the wing (Currer, 2011) and is defined between the chord line and the direction of flight (see Figure 3). This angle influences the pendulum torque around the pitch axis mandatorily and can be controlled by the pilot via the brakes. The negative angle of glide $-\beta$ equals the angle

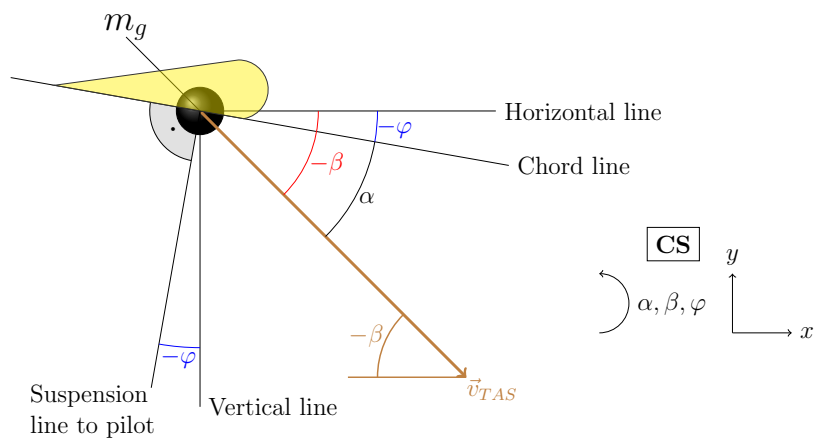

Figure 3. Definition of angles.

of attack $\alpha$ minus the angle of twist $\varphi$. Consequently, the angle of attack $\alpha$ is the difference of the angle of twist $\varphi$ and the angle of glide $\beta$ (see Figure 3 ).

$$
\begin{array}{r}
-\beta=\alpha-\varphi \\
\alpha=\varphi-\beta
\end{array}
$$

The negative angle of twist $-\varphi$ can also be found between the vertical line and the suspension line to the pilot. This information helps to determine the lever arms needed for the torque calculations (see section 2.4).

\subsection{Lever Arms}

As a next step the lever arms of the pendulum system have to be determined. Figure 4 clarifies the relationship between the angles of a paraglider (section 2.3) and the corresponding lever arms (section 2.4). The distances $l_{G m}$ and $l_{M G}$ result from the position of the gravity point $G$ which

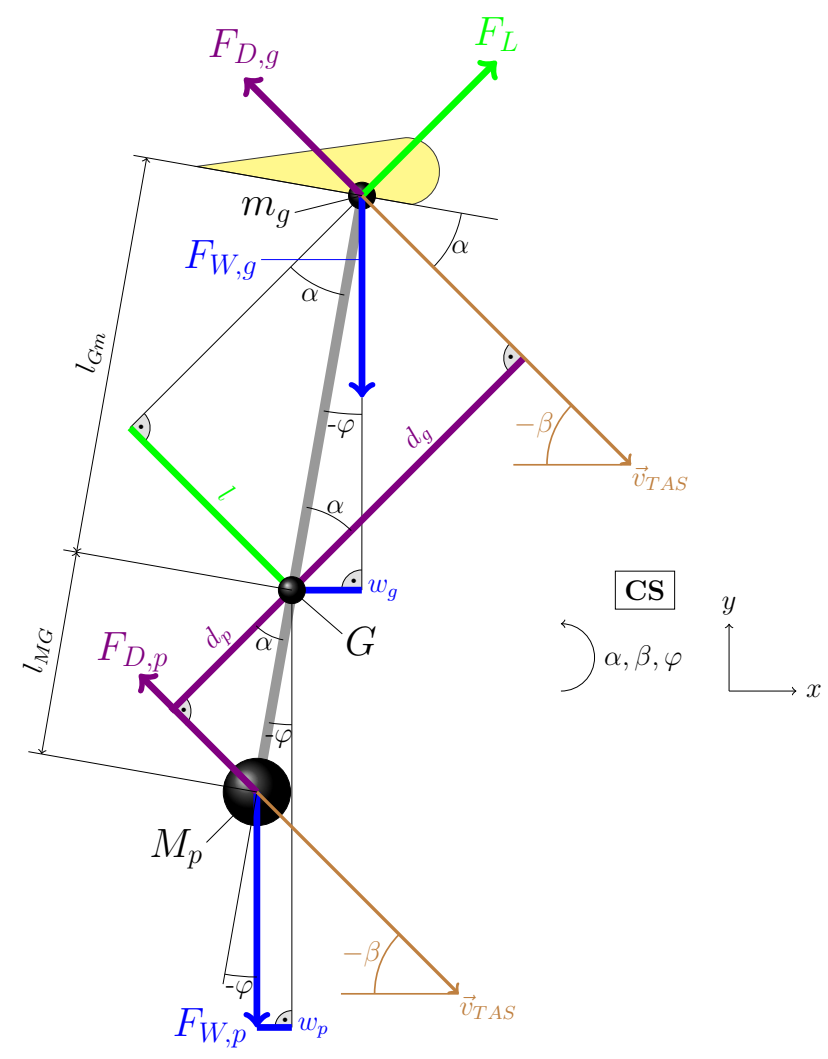

Figure 4. Lever arms.

has been calculated before in section 2.1.

$$
\begin{aligned}
w_{g} & =l_{G m} \cdot \sin (-\varphi) \\
d_{g} & =l_{G m} \cdot \cos (\alpha) \\
l & =l_{G m} \cdot \sin (\alpha) \\
d_{p} & =l_{M G} \cdot \cos (\alpha) \\
w_{p} & =l_{M G} \cdot \sin (-\varphi)
\end{aligned}
$$

With the help of the lever arms the torques around the gravity point $G$ can be determined (see section 2.6).

\subsection{Catenary}

If the pilot starts from the ground and is pulled into the air with the help of the towline, the situation slightly changes.

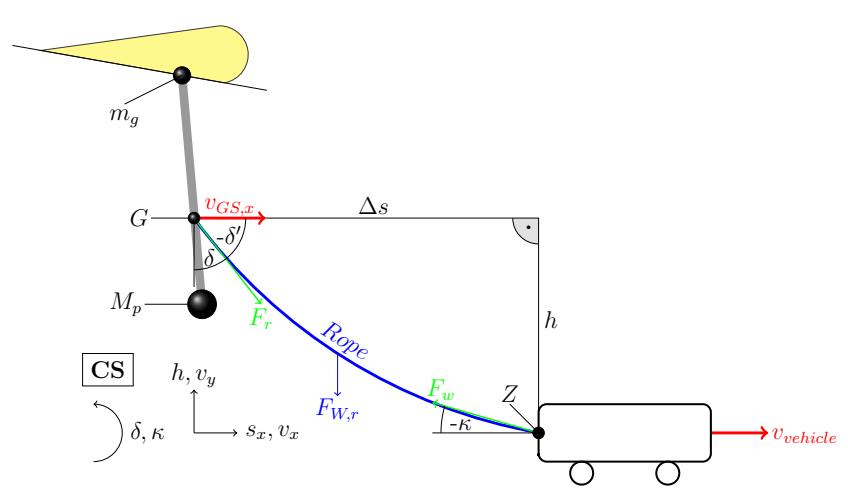

Figure 5. Catenary. 
The weight $F_{W, r}$ of the rope causes a sag and results in a curved line, which directly affects the rope angles $\delta$ and $\kappa$ (see Figure 5). The angles $\delta$ and $\kappa$ are calculated recursively with the help of the catenary. The pilot starts from the ground and thus, the angle $\kappa$ is at the beginning of the towing process zero $\left(\kappa_{\text {initial }}=0^{\circ}\right)$. The catenary can be approximated by the following equation (Dankert and Dankert, 2009)

$$
y(x)=C_{2} \cdot x^{2}+C_{1} \cdot x+C_{0}
$$

where

$$
C_{2}=\frac{0.5 \cdot q_{0}}{F_{w} \cdot \cos (-\kappa)} .
$$

The unknown parameters $C_{0}$ and $C_{1}$ are determined with the help of Figure 5. The height of the catenary at the position $x=0$ equals

$$
y(0 \mathrm{~m})=C_{0}=h
$$

and at the position $x=\Delta s$

$$
\begin{aligned}
& y(\Delta s)=C_{2} \cdot \Delta s^{2}+C_{1} \cdot \Delta s+C_{0}=0 \\
& \Leftrightarrow C_{1}=\frac{-C_{0}}{\Delta s}-C_{2} \cdot \Delta s .
\end{aligned}
$$

Consequently the parameters $C_{1}$ and $C_{2}$ are defined. With the parameters $C_{0}, C_{1}$ and $C_{2}$ the rope angles $\delta$ and $\kappa$ can be calculated. By differentiating equation (20) with respect to $x$ results

$$
\frac{\mathrm{d} y(x)}{\mathrm{d} x}=2 \cdot C_{2} \cdot x+C_{1} .
$$

Figure 5 also displays the derivation at the position $x=0$

$$
\left.\frac{\mathrm{d} y(x)}{\mathrm{d} x}\right|_{x=0}=C_{1}=\tan \left(\delta^{\prime}\right)
$$

with $\delta^{\prime}=\delta-\frac{\pi}{2}$ follows

$$
\delta=\tan ^{-1}\left(C_{1}\right)+\frac{\pi}{2}
$$

as well as at the position $x=\Delta s$

$$
\begin{gathered}
\left.\frac{\mathrm{d} y(x)}{\mathrm{d} x}\right|_{x=\Delta s}=2 \cdot C_{2} \cdot \Delta s+C_{1}=\tan (-\kappa) \\
\Leftrightarrow \kappa=-\tan \left(2 \cdot C_{2} \cdot \Delta s+C_{1}\right) .
\end{gathered}
$$

By balancing the forces in x-direction (see Figure 5) results the rope force $F_{r}$ which is needed for the following section 2.6.

$$
F_{r}=F_{w} \cdot \frac{\cos (-\kappa)}{\sin (\delta)}
$$

Until an altitude of 50 metres the constant winch force $F_{w}$ is reduced to prevent large angles of attack $\alpha$ which could otherwise lead to dangerous flying situations. Safety is very important and gets top priority.

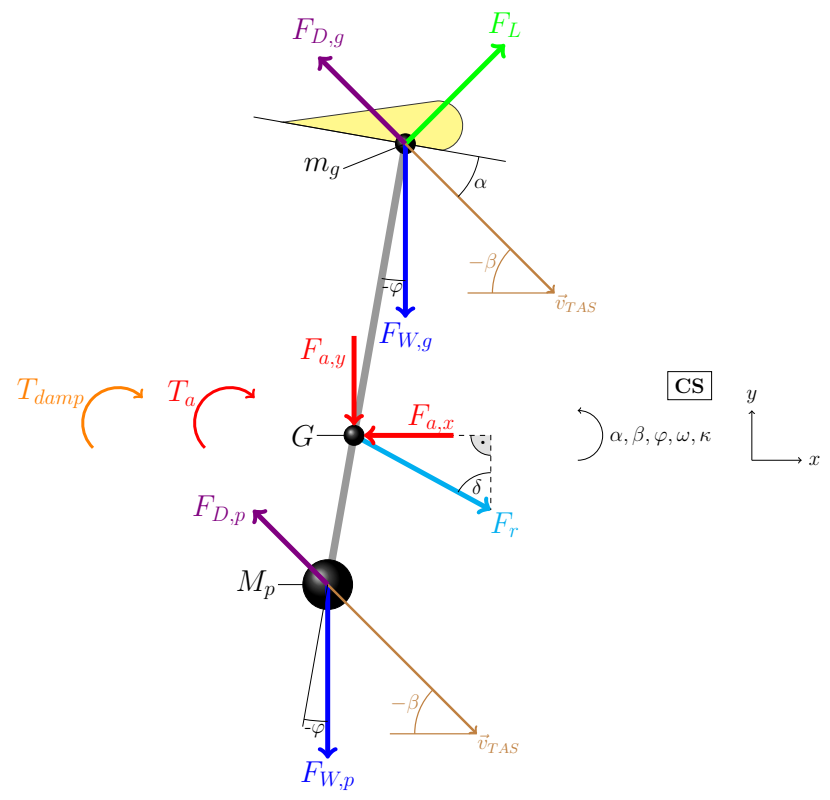

Figure 6. Balance of forces whilst towing.

\subsection{Equations of Motion}

Figure 6 displays the flight system whilst towing. For a gliding flight the rope force $F_{\text {rope }}$ is zero. The algebraic sum of all forces in $\mathrm{x}$-direction is zero. The acceleration force in $\mathrm{x}$-direction equals the total mass $m_{t}$ multiplied by the time derivative of the ground speed in $\mathrm{x}$-direction $\dot{v}_{G S, x}$.

$$
\begin{aligned}
m_{t} \cdot \dot{v}_{G S, x}= & -F_{D, g} \cdot \cos (-\beta)-F_{D, p} \cdot \cos (-\beta) \\
& +F_{L} \cdot \sin (-\beta)+F_{r} \cdot \sin (\delta)
\end{aligned}
$$

Balancing the forces in y-direction results in the following differential equation.

$$
\begin{aligned}
m_{t} \cdot \dot{v}_{G S, y}= & -F_{W, p}-F_{W, g}+F_{D, g} \cdot \sin (-\beta) \\
& +F_{D, p} \cdot \sin (-\beta)+F_{L} \cdot \cos (-\beta) \\
& -F_{r} \cdot \cos (\delta)
\end{aligned}
$$

The algebraic sum of all torques around the gravity point $G$ is zero. The lever arms have already been calculated in section 2.4 and are now used in equation (33). The accelerating torque around the gravity point $G$ equals the total moment of inertia $I_{t}$ multiplied by the time derivative of the angular velocity $\dot{\omega}$.

$$
\begin{aligned}
I_{t} \cdot \dot{\omega}= & +F_{W, p} \cdot w_{p}-F_{W, g} \cdot w_{g}-F_{D, p} \cdot d_{p} \\
& +F_{D, g} \cdot d_{g}-F_{L} \cdot l-T_{d a m p}
\end{aligned}
$$

The damping torque $T_{\text {damp }}$ caused by the movement of the paraglider in the air equals the damping coefficient $d$ multiplied by the angular velocity $\omega$.

$$
T_{\text {damp }}=d \cdot \omega
$$




\subsection{Velocities}

The true air speed $v_{T A S}$ is crucial to calculate the aerodynamic forces. It is the velocity of the airflow in relation to the aerofoil. In addition, the ground speed $v_{G S}$ can be calculated with the true airspeed and the wind speed (Currer, 2011). The true air speed in $\mathrm{x}$-direction $v_{T A S, x}$ is equal to the ground speed in $\mathrm{x}$-direction $v_{G S, x}$ minus the headwind speed $v_{\text {wind } x}$ (equation (35)). The true air speed in y-direction $v_{T A S, y}$ is equivalent to the ground speed in $\mathrm{y}-$ direction $v_{G S, y}$ minus the upwind speed $v_{\text {wind,y }}$ (equation (36)).

$$
\begin{aligned}
& v_{T A S, x}=v_{G S, x}-v_{\text {wind }, x} \\
& v_{T A S, y}=v_{G S, y}-v_{\text {wind }, y}
\end{aligned}
$$

\subsection{Self Stabilisation}
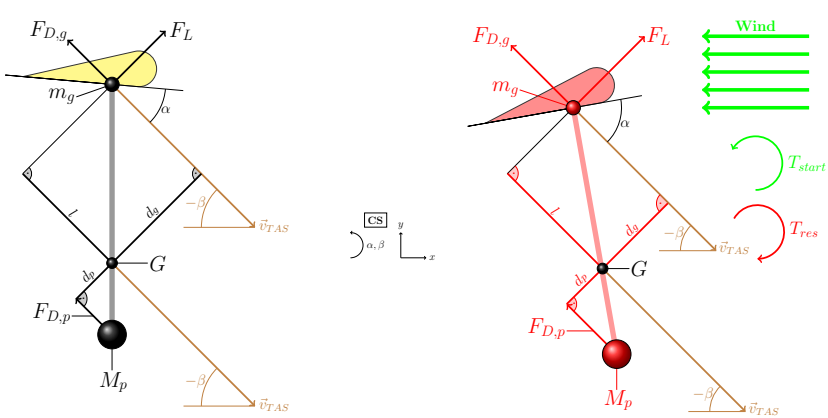

Figure 7. Self stabilisation.

A paraglider is sometimes exposed to turbulence conditions during a flight for example a sudden appearance of contrary wind (see Figure 7). The wind situation at higher altitudes is often different from the situation at ground. It is important to mention in this context that the torques due to weights cancel each other out if the paragliding system starts to swing (see equation (1)). Consequently, the weights $F_{W, g}$ and $F_{W, p}$ are not drawn in Figure 7 . It displays a "standard" flight situation on the left and the reaction of the system due to headwind on the right side. This wind causes a counterclockwise torque $T_{\text {start }}$, which leads to an increase the angle of attack $\alpha$. This affects lift and drag coefficients which are getting larger (see Figure 8). As a result, $F_{L}$ and $F_{D, g}$ are also getting larger and the lever arms $d_{g}$ and $d_{p}$ shorter (see section 2.4). The only increased lever arm is $l$, which leads to an increase of $T_{L}$. This causes a self-aligning moment $T_{\text {res }}$ trying to compensate the start torque. Consequently, the paraglider stabilises on its own and the pilot often does not have to intervene.

\subsection{Complete Model}

Now we summarize our discussion of previous sections and write the complete model of the system. The dynamics of six state variables of the system can be described by the following state equations.

$$
\begin{aligned}
\dot{s}_{x}= & v_{x} \\
\dot{v}_{x}= & \frac{1}{m_{t}} \cdot\left[-F_{D, g} \cdot \cos (-\beta)-F_{D, p} \cdot \cos (-\beta)\right. \\
& \left.+F_{L} \cdot \sin (-\beta)+F_{r} \cdot \sin (\delta)\right] \\
\dot{s}_{y}= & v_{y} \\
\dot{v}_{y}= & \frac{1}{m_{t}} \cdot\left[-F_{W, p}-F_{W, g}+F_{D, g} \cdot \sin (-\beta)\right. \\
& \left.+F_{D, p} \cdot \sin (-\beta)+F_{L} \cdot \cos (-\beta)-F_{r} \cdot \cos (\delta)\right] \\
\dot{\varphi}= & \omega \\
\dot{\omega}= & \frac{1}{I_{t}} \cdot\left[+F_{W, p} \cdot w_{p}-F_{W, g} \cdot w_{g}-F_{D, p} \cdot d_{p}\right. \\
& \left.+F_{D, g} \cdot d_{g}-F_{L} \cdot l-d \cdot \omega\right]
\end{aligned}
$$

With

$$
\begin{aligned}
F_{L} & =c_{l} \cdot A_{\text {project }} \cdot \frac{\rho_{\text {air }}}{2} \cdot\left(v_{T A S}\right)^{2} \\
F_{D, g} & =c_{d, g} \cdot A_{\text {project }} \cdot \frac{\rho_{\text {air }}}{2} \cdot\left(v_{T A S}\right)^{2} \\
F_{D, p} & =c_{d, p} \cdot A_{\text {pilot }} \cdot \frac{\rho_{\text {air }}}{2} \cdot\left(v_{T A S}\right)^{2} \\
F_{W, g} & =m_{g} \cdot g \text { and } F_{W, p}=M_{p} \cdot g \\
F_{D, p} & =c_{d, p} \cdot A_{\text {project }} \cdot \frac{\rho_{\text {air }}}{2} \cdot\left(v_{T A S}\right)^{2} \\
v_{T A S, x} & =v_{G S, x}-v_{\text {wind }, x} \\
v_{T A S, y} & =v_{G S, y}-v_{\text {wind }, y} \\
\beta & =\arctan \left(\frac{v_{T A S, y}}{v_{T A S, x}}\right) \\
\alpha & =\varphi-\beta \\
\delta & =\tan ^{-1}\left(C_{1}\right)+\frac{\pi}{2}
\end{aligned}
$$

\section{Model Parameters}

In this section we want to summarize important parameters used in this model. Values of some of the parameters are provided by the manufacturer or calculated analytically. Other parameters are tuned experimentally, for instant the damping coefficient $d$, to get a good image of the reality.

$$
\begin{array}{ll}
\rho_{\text {air }}=1.27 \frac{\mathrm{kg}}{\mathrm{m}^{3}} & m_{g}=6 \mathrm{~kg} \\
M_{p}=100 \mathrm{~kg} & l_{\text {line }}=6.97 \mathrm{~m} \\
A_{\text {projected }}=24.26 \mathrm{~m}^{2} & A_{\text {pilot }}=1.0 \mathrm{~m}^{2} \\
l_{M G}=0.395 \mathrm{~m} & l_{\text {Gm }}=6.575 \mathrm{~m} \\
d=10000 \frac{\mathrm{Nms}}{\mathrm{rad}} & c_{d, p}=0.33
\end{array}
$$

The aerodynamic coefficients $c_{l}$ and $c_{d, g}$ are assumed to be functions of the angle of attack $\alpha$. These dependencies are shown in Figure 8 graphically. 


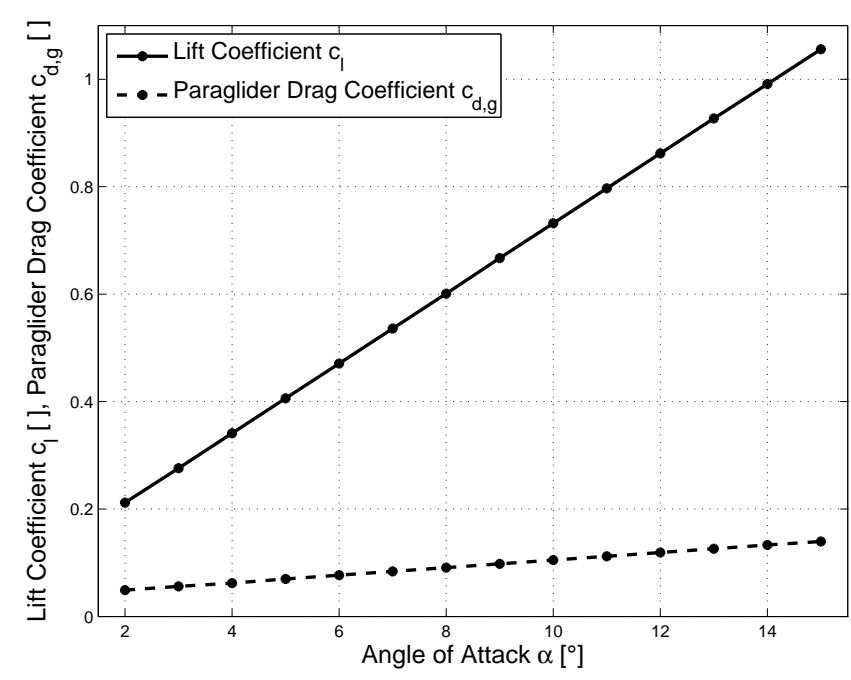

Figure 8. Aerodynamic coefficients as functions of $\alpha$.

\section{Simulation Results}

The model is implemented in Matlab/Simulink. Two different flight situations are simulated. The first one is a gliding flight, where the pilot begins to fly at a certain height. The second one extends the first model by a winch launch to enable the pilot to gain altitude.

\subsection{Gliding Flight}
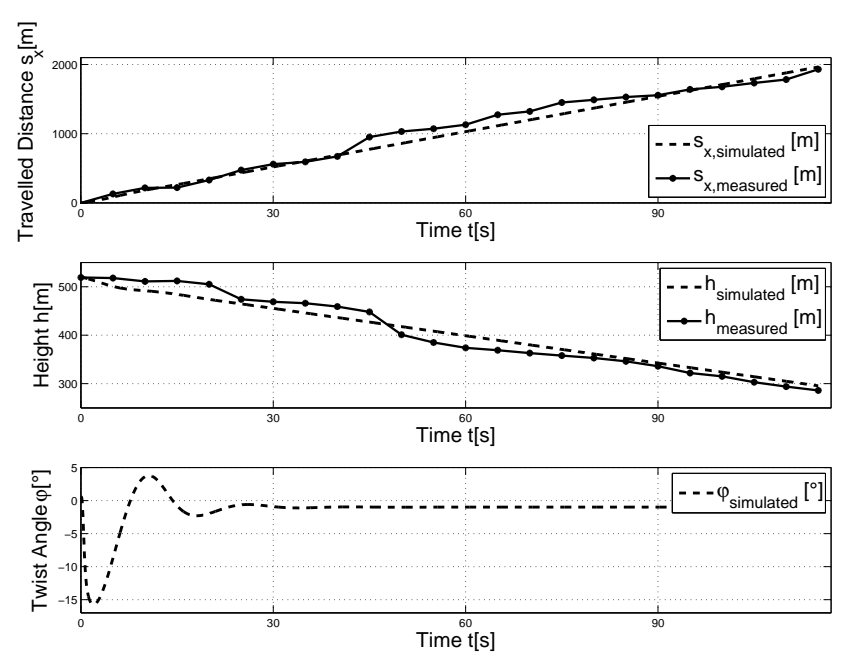

Figure 9. Gliding flight.

The three plots seen on Figure 9 show the translational motion of the system over the time. The angle of glide $\beta$ is constant during the whole flight. With a start height $h$ of 500 metres, a flight distance in x-direction $s_{x}$ of $1930 \mathrm{~m}$ is reached on the expense of a $230 \mathrm{~m}$ loss in altitude. The third plot illustrates the dynamics of the twist angle $\varphi$ with a settling time of about $30 \mathrm{~s}$. The deviation between the simulated and measured results may be due to different wind situations. The wind affects the system behaviour significantly (see Figure 10). At the beginning,
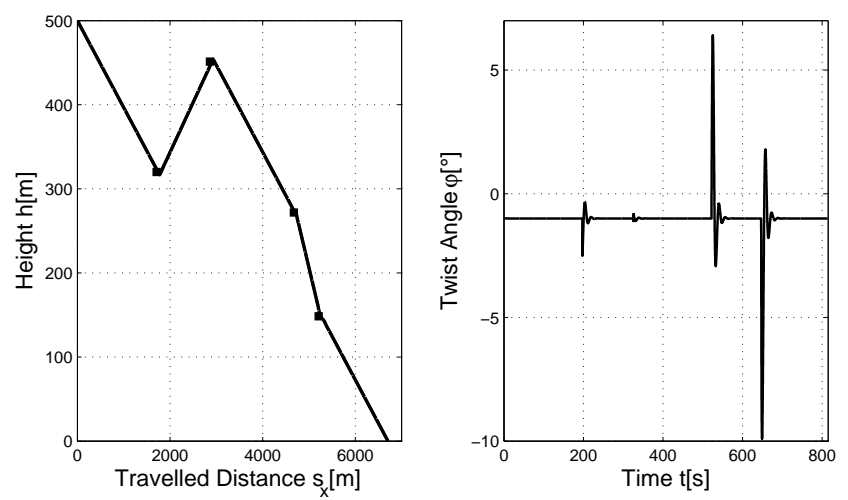

Figure 10. Gliding flight with wind impact.

the pilot glides down and is slowly reducing altitude. Subsequently, the pilot enters an updraft region (first black square) and, as a result, the pilot gains altitude and rises up to $450 \mathrm{~m}$ (second black square), where the helpful wind stops. The pilot glides down to the third black square, where headwind prevails. Consequently, the ground speed $v_{G S}$ reduces and the angle of glide $\beta$ increases. Therefore, height is lost more quickly than before and, as a result, the travelled flight distance $s_{x}$ gets shorter. At a height of $150 \mathrm{~m}$ (fourth black square), the headwind levels off and the paraglider flies again with trim speed. The reaction of the twist angle $\varphi$ in relation to the changing conditions is displayed on the right side of Figure 10. If the ground speed $v_{G S}$ changes due to the impact of the wind, the angle of twist $\varphi$ is disturbed from its steady-state value and after short time it is settled again. The disturbance caused by horizontal wind is stronger than the vertical wind, because headwind or tailwind directly creates a torque around the gravity point $G$ and deflects the system from the rest position.

\subsection{Towing Process}
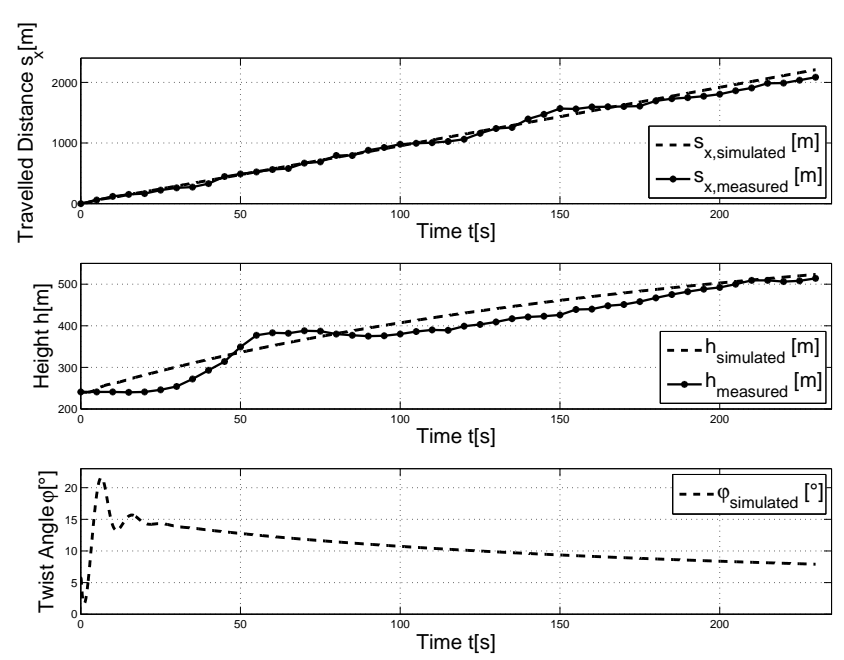

Figure 11. Towing process. 
The first diagram of Figure 11 shows the travelled distance of the paraglider in X-direction $s_{x}$ over the time. It is easy to recognize that the movement is linear due to the constant slope of the graph. The second plot shows the height $h$, whereas the transient response of the twist angle $\varphi$ is drawn in the last diagram of Figure 11. A comparison of measured and simulated results reveals that the simulation model provides a fair reflection of the reality.

\section{Conclusions}

A simulation model for a paraglider is derived and implemented in Matlab/Simulink. The parameters are adjusted in order to simulate the behaviour of a certain paraglider. With the help of the model two different flight situations, i.e. gliding flight and towing process, are simulated. Simulation results are compared with data of a real flight. The model provides a good approximation of the dynamical behaviour of the real system. It allows to compare different parameter settings, determine their influence on the system as well as to optimise the towing process. However, as already mentioned, paragliding is under constant change and this model has to be adjusted accordingly.

\section{References}

J. D. Anderson. Introduction to Flight. McGraw-Hill, New York, 5th edition, 2005.

I. Currer. Touching Cloudbase - The Complete Guide to Paragliding. Air Supplies, York, 5th edition, 2011.

J. Dankert and H. Dankert. Technische Mechanik: Statik, Festigkeitslehre, Kinematik/Kinetik. Vieweg+Teubner, Wiesbaden, 5th edition, 2009.

H. Fahr. Gleitsegelschlepp. Flieg Zeug, Magdeburg, 2nd edition, 1992.

P. Janssen, K. Slezak, and K. Tänzler. Gleitschirmfliegen: Theorie und Praxis. Nymphenburger, München, 18th edition, 2013.

F. Karbstein. Richtig Paragliding. BLV Verlagsgesellschaft, München, 1996.

H. Oertel. Introduction to Fluid Mechanics: Fundamentals and Applications. Universitätsverlag, Karlsruhe, 2005.

O. Voigt. Aerodynamik und Flugmechanik des Gleitschirms. Books on Demand (Schweiz), Norderstedt, 2nd edition, 2003.

N. Whittall. Paragliding: The Complete Guide. Lyons Press Series. Lyons Press, Lanham, 1995.

N. Whittall. Hang Gliding \& Paragliding. Clash series. Ticktock Media, Kent, 2010. 